\title{
Uncovering the relationship and mechanisms of Tartary buckwheat (Fagopyrum tataricum) and Type II diabetes, hypertension, and hyperlipidemia using a network pharmacology approach
}

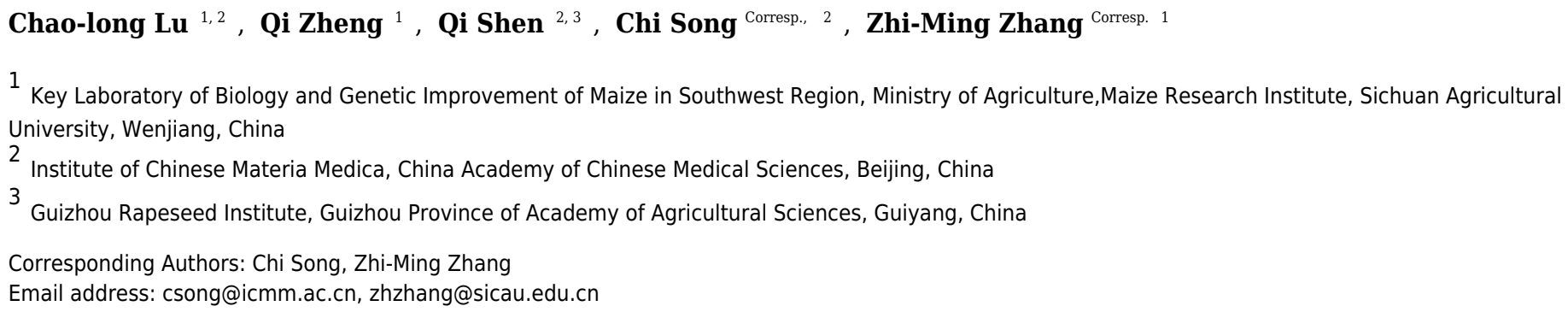

Background: Tartary buckwheat (TB), a crop rich in protein, dietary fiber, and flavonoids, has been reported to have an effect on Type II diabetes (T2D), hypertension (HT), and hyperlipidemia (HL). However, limited information is available about the relationship between Tartary buckwheat and these three diseases. The mechanisms of how TB impacts these diseases are still unclear.

Methods: In this study, network pharmacology was used to investigate the relationship between the herb as well as the diseases and the mechanisms of how TB might impact these diseases.

Results: A total of 97 putative targets of 20 compounds found in TB were obtained. Then, an interaction network of 97 putative targets for these compounds and known therapeutic targets for the treatment of the three diseases was constructed. Based on the constructed network, 28 major nodes were identified as the key targets of TB due to their importance in network topology. The targets of ATK2, IKBKB, RAF1, CHUK, TNF, JUN, and PRKCA were mainly involved in fluid shear stress and the atherosclerosis and PI3KAkt signaling pathways. Finally, molecular docking simulation showed that 174 pairs of chemical components and the corresponding key targets had strong binding efficiencies.

Conclusion: For the first time, a comprehensive systemic approach integrating drug target prediction, network analysis, and molecular docking simulation was developed to reveal the relationships and mechanisms between the putative targets in TB and T2D, HT, and HL. 


\section{Uncovering the relationship and mechanisms of Tartary}

\section{2 buckwheat (Fagopyrum tataricum) and Type II diabetes,}

\section{3 hypertension, and hyperlipidemia using a network}

\section{4 pharmacology approach}

5 Chao-Long Lu 1,3, Qi Zheng ${ }^{1}$, Qi Shen ${ }^{2,3}$, Chi Song ${ }^{3 *}$ and Zhi-Ming Zhang ${ }^{1, *}$

6 'Key Laboratory of Biology and Genetic Improvement of Maize in Southwest Region, Ministry of Agriculture,

7 Maize Research Institute of Sichuan Agricultural University, Wenjiang, Sichuan, 611130, China

$8{ }^{2}$ Guizhou Rapeseed Institute, Guizhou Province of Academy of Agricultural Sciences, Guiyang, 550025, China

$9{ }^{3}$ Institute of Chinese Materia Medica, China Academy of Chinese Medical Sciences, Beijing, 100700, China

10

$11 *$ Correspondence author:

12 Zhi-Ming Zhang

13 Key Laboratory of Biology and Genetic Improvement of Maize in Southwest Region, Ministry of Agriculture,

14 Maize Research Institute of Sichuan Agricultural University, Wenjiang, Sichuan, 611130, China

15 Email address: zhzhang@sicau.edu.cn

16 or

17 Chi Song

18 Institute of Chinese Materia Medica, China Academy of Chinese Medical Sciences, Beijing, 100700, China

19 Email address: csong@icmm.ac.cn (Chi Song) 


\section{Abstract}

Background: Tartary buckwheat (TB), a crop rich in protein, dietary fiber, and flavonoids, has been reported to have an effect on Type II diabetes (T2D), hypertension (HT), and hyperlipidemia (HL). However, limited information is available about the relationship between Tartary buckwheat and these three diseases. The mechanisms of how TB impacts these diseases are still unclear.

Methods: In this study, network pharmacology was used to investigate the relationship between the herb as well as the diseases and the mechanisms of how TB might impact these diseases.

Results: A total of 97 putative targets of 20 compounds found in TB were obtained. Then, an interaction network of 97 putative targets for these compounds and known therapeutic targets for the treatment of the three diseases was constructed. Based on the constructed network, 28 major nodes were identified as the key targets of TB due to their importance in network topology. The targets of ATK2, IKBKB, RAF1, CHUK, TNF, JUN, and PRKCA were mainly involved in fluid shear stress and the atherosclerosis and PI3K-Akt signaling pathways. Finally, molecular docking simulation showed that 174 pairs of chemical components and the corresponding key targets had strong binding efficiencies.

Conclusion: For the first time, a comprehensive systemic approach integrating drug target prediction, network analysis, and molecular docking simulation was developed to reveal the relationships and mechanisms between the putative targets in TB and T2D, HT, and HL.

\section{Introduction}

Tartary buckwheat (TB; Fagopyrum tataricum) is widely distributed in the temperate zones of the Northern Hemisphere in countries that include: China, Europe, North America, Korea, and Japan (Campbell 1997; Ohsako T 2002). TB is a medicinal and edible crop that is rich in carbohydrates, flavonoids, and chemical compounds, thus it can be used to prevent cardiovascular diseases and diabetes because of its high nutritive value and special effect on physiological regulation (Fabjan et al. 2003; Kreft 2016; Lin 1994; Wieslander 1996). The rutin content in TB seed is approximately 100 times $(0.8-1.7 \%)$ higher than that in common buckwheat $(F$. esculentum) (0.01\%) (Fabjan et al. 2003). The earliest record of the medical function of TB in Chinese history traces back to about 2,000 years ago (Lin 1994). However it has only been in recent years that TB, a health-beneficial crop, has attracted a large attention for its nutraceutical functions (Kreft 2016; Prakash \& Deshwal 2013). 
Type II diabetes (T2D), hypertension (HT) and hyperlipidemia (HL) are three major diseases with a high incidence in modern society, which have seriously damaged human health. TB has been reported to have the ability to decrease the risk of type 2 diabetes mellitus (T2DM) (Lee et al. 2012; Zhang et al. 2012); research on TB has indicated that dietary Tartary buckwheat intake attenuates insulin resistance and improves lipid profiles in patients with T2D (Qiu et al. 2016b). A diet that includes TB can also reduce the blood sugar levels of patients with T2D, demonstrating that TB can contribute to the effective control of T2D (Lee et al. 2016; Zhou et al. 2015). Moreover, TB is able to antagonize the increase of capillary fragility associated with hypertension in humans (Jisoon Im et al. 2003; Kreft et al. 1999). Ethanol extract from buckwheat, rutin, and quercetin have been proven to boost Akt phosphorylation and interrupt PPAR $\gamma$ degradation in the hepatocyte cell line, leading to improved glucose uptake (Lee et al. 2012). TB rutin-free extracts likely mediate the NO/cGMP pathways, thereby exerting endothelium-dependent vasorelaxation action (Ushida et al. 2008). The endogenous vasodilators bradykinin and NO were upregulated by TB sprouts, and, together with a lower level of the vasoconstrictor endothelin-1, relieve hypertension and oxidative stress in vivo (Merendino et al. 2014). In addition, Tartary buckwheat shell extract (TBSE) resists hyperlipidemia (Tong et al. 2006). Based on an assay used in rats fed a high-fat diet, apparent reductions in weight gain, plasma lipid concentrations, and atherogenic index were found in those rats with diets supplemented with buckwheat leaf and flowers compared with those that received no supplementation, demonstrating that buckwheat products are potential prevention and curing agents of hyperlipidemia (Brenesel et al. 2013). Although TB has been well-practiced in clinical medicine, the fundamental mechanisms and relationships between TB compounds and the interaction of these three diseases remain elusive.

TB has been demonstrated that insulin resistance was attenuated and that lipid profiles was also ameliorated in patients with T2D after dieting TB (>110 g/d) for 4 weeks (Qiu et al. 2016a). A research on $75 \%$ ethanol extract of TB (EETB) and rutin demonstrated that both EETB and rutin suppresses the formation of fructosamine and $\alpha$-dicarbonyl compounds to lower the level of AGEs (advanced glycation end-products). Hence, EETB can be considered as a potential protective agent for diabetic patients (Lee et al. 2015).

In the past few years there has been a considerable interest in the improvement of diabetic control to alter the glycemic impact on carbohydrates intake. A low glycemic index (GI) diet has been related to advantages in the prevention and treatment of diabetes (Ajala et al. 2013). Skrabanja et al. demonstrated the benefit of TB to reduce the plasma glucose. When 10 healthy testers consumed different single dose diets, boiled TB groats, bread with 50\% TB groats and white wheat bread, the postpandrial plasma glucose and insulin level were tested. The results showed those who consumed TB products or groats had lower plasma glucose and insulin level compared with those who have white wheat bread (Vida Skrabanja et al. 2001). Su-Que et al reached the same conclusion from a different aspect. 10 T2D patients were randomly selected to consume TB or white wheat bread, and the postpandrial $2 \mathrm{~h}$ plasma glucose in the subjects having TB showed a 
92 decrease of $51 \%(\mathrm{p}<0.05)$ compared that in those have white wheat bread (Lan et al. 2013). Thus,

93 TB will be a potential treatment to reduce the risk for T2D and HT.

94 With the development of system biology, network biology, and polypharmacology came the 95 concept of network pharmacology, which was first proposed by Andrew L. Hopkins and is based 96 on the application of multiomics and systemic biological technology (Hopkins 2007). Its aim is to 97 discover the synergistic effects and potential mechanisms of interaction between multi98 components and targets by analyzing complex and multilevel interactive networks. Network pharmacology is widely used in drug discovery, target prediction, and mechanism research, especially in traditional herbal medicine (Jiansheng Li 2015; Zhang et al. 2016). This article applies network pharmacology to investigate the mechanism of TB and its interaction with T2D, hypertension, and hyperlipidemia at the target level. Our study provides a comprehensive view of the relationships and mechanisms between TB and T2D, HT, and HL.

\section{Materials and methods}

\section{Composite compounds of Tartary buckwheat}

We collected the composite compound data of Tartary buckwheat from the Universal Natural Products Database (UNPD) (Gu et al. 2013) (http://pkuxxj.pku.edu.cn/UNPD/, updated April 25 2013), which was specifically designed to store natural product structures for drug discovery and network pharmacology. In total, the structural information of 20 Fagopyrum tataricum compounds was collected. Detailed information on the composite compounds of Tartary buckwheat is provided in Supplementary Table S1.

\section{Known therapeutic targets of diseases}

The known therapeutic target data for the treatment of T2D, HT, and HL were collected from two resources: DrugBank (Law 2014) (http://www.drugbank.ca/, version 4.0) and Online Mendelian Inheritance in Man (OMIM) (Scott et al. 2000) (http://www.omim.org/, last accessed: October 31, 2015). In the DrugBank database, the targets were collected based on the following criteria: (1) they were FDA-approved therapeutic targets of the three diseases; and (2) the targets of drugs were human genes/proteins. In the OMIM database, we used the keywords "Type 2 diabetes," "hypertension," and "hyperlipidemia" as the queries to search known therapeutic targets of diseases. After removing the redundant results, there were 59, 279, and 20 known therapeutic targets for the treatment of T2D, HT, and HL, respectively. Detailed information on the therapeutic targets of the diseases is provided in Supplementary Table S2, S3, and S4. 
123

124

125

126

127

128

129

130

131

132

133

134

135

136

137

138

139

140

141

142

143

144

145

146

147

148

149

150

151

152

153

\section{Protein-protein interaction (PPI) data}

PPI data were retrieved from eight public available databases: Biological General Repository for Interaction Datasets (BioGRID) (Stark et al. 2011), Human Annotated and Predicted Protein Interaction Database (HAPPI) (Chen 2009), Human Protein Reference Database (HPRD) (Keshava Prasad et al. 2009), High-quality INTeractomes (HINT) (Jishnu \& Yu 2012), Molecular INTeraction Database (MINT) (Chatraryamontri 2010), Online Predicted Human Interaction Database (OPHID) (Brown \& Jurisica 2005), Database of Interacting Proteins (DIP) (Xenarios et al. 2002), and Search Tool for the Retrieval of Interacting Genes/Proteins (STRING) (Szklarczyk et al. 2011). Detailed information from the eight databases is provided in Supplementary Table S5.

\section{Target prediction of composite compounds}

The Bioinformatics Analysis Tool for Molecular mechANism of Traditional Chinese Medicine (BATMAN-TCM) database (Liu et al. 2016b), which is aimed at the target prediction of composite compounds of tartar buckwheat, was employed. In this database, there are 6 basal principles for the measurement of drug-drug similarity that are based on chemical structure (including FP2 fingerprint-based and functional group-based similarity scores), side effects, the Anatomical, Therapeutic and Chemical (ATC) classification system, drug-induced gene expression, and the text mining score of chemical-chemical associations, and 3 scores to measure protein-protein similarity respectively based on protein sequence, closeness in a protein interaction network and Gene Ontology (GO) functional annotation. The default parameters were set for the putative targets of composite compounds of Tartary buckwheat.

\section{Network construction and analysis}

The TB-composite compound-putative target-known therapeutic target network was constructed to find the key target. Then, the target-pathway network was established to find the relationship between the pathways and the key targets. The key target-pathway networks would be used to explore core pathways that could play an important role in the interaction mechanism of TB and the three diseases.

Cytoscape (Shannon et al. 2003) (http://www.cytoscape.org/, version:3.2.1) and NAViGaTOR (http://ophid.utoronto.ca/navigator/, version:2.3) were employed to directly visualize the networks. In addition, four topological features ('Degree,' 'Betweenness,' 'Closeness,' and 'K core') were calculated using the igraph package, which is a powerful tool for topological graphing in R (https://cran.r-project.org/). 
154

155

156

157

158

159

160

161

162

163

164

165

166

167

168

169

170

171

172

173

174

175

176

177

The networks were simplified using the following procedure: (A) we deleted the nodes that had degree values of less than 2-fold the median of all of the nodes in the network, and we then used the retained nodes to construct the hub network. (B) We retained the nodes that were greater than the corresponding median values of the four topological features: 'Degree,' 'Betweenness,' 'Closeness,' and 'K core' (Li et al. 2007).

\section{Pathway enrichment analyses}

The clusterProfiler package of $\mathrm{R}$ software (Yu et al. 2012) was employed to classify the biological terms and to analyze the gene cluster enrichment automatically. The latest data were obtained from the Kyoto Encyclopedia of Genes and Genomes (KEGG) database (Kanehisa \& Goto 1999) for KEGG pathway enrichment analyses. P-values were set at 0.05 as the cut-off criterion, and the results of both analyses were annotated by Pathview (Luo 2013) in the R Bioconductor package (https://www.bioconductor.org/).

\section{Molecular docking simulation}

LibDock was implemented in the Discovery Studio 2.5 (DS 2.5) software to determine the molecular docking simulation. It is an efficient and powerful tool to validate the binding ability of candidate targets to composite compounds of herbs. All of the crystal structure data of the targets were directly retrieved from the RCSB Protein Data Bank (http://www.rcsb.org/pdb/home/home.do, last accessed Dec 27, 2016). The high-resolution crystal structure was a priority for verification. We then utilized the customizable scoring function from LibDock to calculate the docking score to measure the binding ability of each candidate target of the corresponding compound. The docking scores of the candidate targets with a strong binding ability to their corresponding compounds were greater than the median value of the all of the docking scores.

\section{Results and discussion}

\section{Putative targets for Tartary buckwheat}

A total of 20 ingredients in TB were retrieved from the Universal Natural Products Database (UNPD). The detailed information about these molecules is provided in Supplementary Table S1. Following the drug target predicted by BATMAN-TCM, 97 putative targets of the 20 ingredients of TB were identified (Supplementary Table S6). In addition, known therapeutic targets of the 
183 three diseases were collected from two public databases (described in the Materials and methods

184

185

186

187 section). We obtained 59, 279, and 20 known therapeutic targets for the treatment of T2D, HT, and HL, respectively. Interestingly, 8 and 1 putative targets of TB were significant proteins for HT and HL, respectively (Figure 1). PPARG (Peroxisome proliferator-activated receptor gamma) was shared by PT, T2D, and HT; ABCA1 (ATP-binding cassette sub-family A member 1) was shared by PT, T2D, and HL; PPARA (Peroxisome proliferator-activated receptor alpha) was shared by PT, HT, and HL; and SLC6A4 (Sodium-dependent serotonin transporter) was shared by PT, T2D, HT, and HL (Supplementary Table S7). SLC6A4 plays a significant role in regulating serotonin for the availability of other serotonin system receptors (Comings et al. 1999; Zhang et al. 2007). PPARA is a key regulator of lipid metabolism (Gorla-Bajszczak et al. 1999; Laurent et al. 2013). ABCA1 functions as a key gatekeeper influencing intracellular cholesterol transport (Kathiresan et al. 2008; Singaraja et al. 2003). PPARG is important for its regulation of adipocyte differentiation and retention of glucose homeostasis (Katanotoki et al. 2013; Mukherjee et al. 1997; Park et al. 2011). Out of the 97 putative targets of TB compounds, there were 13 that were related to these three diseases, suggesting the possibility of TB as their treatment.

Figure 1. Venn diagram showing the overlap of significant targets in PT, T2D, HT, and HL. PT=putative targets, green; T2D = type II diabetes, blue; HT=hypertension, pink; and HL=hyperlipidemia, yellow.

\section{Identification of the underlying pharmacological mechanisms of TB}

\section{on the three diseases}

A network was constructed based on TB-composite compound-putative targets and known therapeutic targets of the diseases to elucidate the pharmacological mechanisms of TB on these three diseases. Protein-protein interaction (PPI) data of the putative targets and the known therapeutic targets of the three diseases were collected from eight public PPI databases (as described in the Materials and methods section). The network consisted of 455 nodes and 1748 edges in total. Two-fold the median value of degree was set as the threshold. The network was reconstructed after deleting the nodes that were less than the threshold. As a result, the nodes were reduced from 455 to 132 , and the edges from 1748 to 1010 in the reconstructed network. In order to determine the key targets in the network, four attributes ('Degree,' 'Betweenness,' 'Closeness,' and ' $\mathrm{K}$ core') were calculated in the topological networks. The network was further simplified with these four values, and the key target information was finally obtained. The four topological features were used to retain the nodes that were over the median in the rebuilt network. The median values of 'Degree,' 'Betweenness,' 'Closeness,' and 'K core' were 10.0000, 38.2517, 0.0034, and 8.0000 , respectively. Therefore, targets with 'Degree'>10.0000, 'Betweenness'> 38.2517, 
217 'Closeness' $>0.0034$, and 'K core' $>8.0000$ were defined as the key targets (Supplementary Table

218 S8). As a result, the network that was rebuilt with the key targets had 29 nodes and 163 edges

219 (Figure 2).

Figure 2. Interaction network between chemical components of TB, their putative targets, and known therapeutic targets of the three diseases built and visualized with Cytoscape. Blue line: linked PT and their targets; purple: linked T2D and their targets; green: linked HL and their targets; yellow: linked HT and their targets; and light blue: linked chemical components and their targets.

Lines with different colors were employed to show their importance from the targets to their corresponding sources (PT, T2D, HT, and HL) in our network, and diameter was used to denote degree. A larger node diameter represented a higher degree in the network, and vice versa. Similarly, with the targets, those with the higher degree played a more important role in the network. Compared with all of the other targets, SRC (Proto-oncogene tyrosine-protein kinase Src), JUN, and IL1B (Interleukin-1 beta) had the highest degree number (19), which indicated that these targets play key roles in the regulation of T2D, HT, and HL. Our results agreed well with previous research, demonstrating that JUN modulated smooth muscle cell proliferation in response to vascular angioplasty ( $\mathrm{Hu}$ et al. 1997), SRC modulated endothelial cell angiogenic activities inflammation in obesity (Maldonado-Ruiz et al. 2017; Osborn et al. 2008). Moreover, PT, T2D, HL, and HT were linked to 10, 4, 1, and 17 key targets in the network, respectively. Specifically, UNPD28717 (salicylic acid) was linked to 9 key targets, indicating that it may mediate these targets to regulate blood-vessel dilation, inflammatory cytokine, and adipose tissue (Liu et al. 2016a; Tang \& Dong 2017).

Pathway analysis to explore the underlying mechanisms of TB and

In order to investigate the relationship and mechanisms between the targets and the pathways, a target-pathway network was constructed (as described in the Materials and Methods section). The KEGG database was used to describe KEGG pathways, to systematically analyze gene functions, and to provide a reference knowledge base linking genomes to functional information. In total, 48 pathways were obtained by igraph to analyze the KEGG enrichment of key targets. The pathway-target network contained 76 nodes (48 pathways and 28 targets) and 352 edges. The median values of 'Degree,' 'Betweenness,' 'Closeness,' and 'K core' were 7.0000, 23.3158, 0.0059, and 6.0000, respectively (Supplementary Table S9). AKT2, IKBKB, RAF1, TNF, and CHUK were in the top-ranking positions in the pathway-target network. Additionally, the results 
251 indicated that some targets had been hit by multiple pathways in the pathway-target network.

252

253

254

255

256

257

258

259

260

261

262

263

264

265

266

267

268

269

270

271

272

273

274

275

276

277

278

279

280

281

282

283

284

285

ATK2, IKBKB, RAF1, CHUK, TNF, JUN, and PRKCA were linked by 42, 32, 29, 26, 26, 24, and 17 pathways (Figure 3). AKT2 (RAC-beta serine/threonine-protein kinase) is responsible for the regulation of glucose uptake by mediating insulin-induced translocation (Hers et al. 2011; Zhang et al. 2006). IKBKB (inhibitor of nuclear factor kappa-B kinase subunit beta) plays an essential role in the NF-kappa-B signaling pathway (hsa04064), which is activated by multiple stimuli, such as inflammatory cytokines (Mercurio et al. 1997). RAF1 (RAF proto-oncogene serine/threonineprotein kinase) is involved in proliferation and angiogenesis (Chong et al. 2001; Yao et al. 1995).

Pathways related to these targets were shown to have more significant features (Figure 3). Among the pathways, hsa5200 (a pathway in cancer) exhibited the highest number of target connections (degree=15), followed by hsa05418 (a fluid shear stress and atherosclerosis pathway) with 14 targets, and hsa04151 (a PI3K-Akt signaling pathway) with 11 targets, respectively. These high-degree pathways were closely related to vascular conditions and inflammation. The hsa5200 pathway was the underlying mechanism of inflammation and involved in multiple targets, such as PPARG, JUN, CHUK, IKBKB, AKT2, and RAF1 (Andersen et al. 2017; Kolb et al. 2016). The fluid shear stress and atherosclerosis pathway plays an important role in atherosclerosis, and it is associated with systemic risk factors, including hypertension, hyperlipidemia, and diabetes mellitus (Cheng et al. 2006; Malek et al. 1999). The PI3K-Akt signaling pathway is one of the best characterized downstream effectors of insulin and belongs to insulin-activated intracellular pathways (Westermeier et al. 2011). In addition, we found that some pathways discovered in this study, such as the insulin resistance pathway, AGE-RAGE (Advanced glycation end products) signaling pathway (Hegab et al. 2012; Roy 2013), and insulin signaling pathway (Supplementary Table S10), have a direct relationship with the three diseases. Overall, the key targets are significantly associated with these pathways that might play a role in the progression of the three diseases.

Figure 3. The target-pathway network. Pink dots are targets, purple diamonds are pathways, and the dot size and diamond size represent node degree value.

\section{Molecular docking validation}

The computational docking technique, as a structure-based method, is an invaluable tool in drug discovery and design. This technique can help researchers discover the relationships between the constituents of TCM and network targets(Luo et al. 2014a; Luo et al. 2014b; Yu et al. 2016). The Libdock module of the DS2.5 software was used for molecular analog docking to obtain the effective dockings of TB and its key targets and to get docking scores. The score was greater than the median value (86), indicating a strong binding capacity between the composite components of 
286 TB and the molecular targets in this study. In total, we obtained 174 docking results. Among these

287 results, JUN received the highest score of 170.967 with chemical UNPD51223 (Supplementary

288 Table S11). The docking score results were used to construct the target-composite component

289 network (Figure 4). The target-composite compound network contains 38 nodes (21 targets and 17

290 composite compounds) and 174 edges. In addition, the line width shows the docking value,

291 meaning a thicker line represents a higher docking value and vice versa. As a result, JUN, TNF,

292 PPARA, PPP2CA, PPARG, and IKBKB had a high degree value and larger molecular analog 293 docking scores (Supplementary Table S12). These targets were proven to bind to multiple 294 chemicals.

Figure 4. The target-composite compound network. Blue dots are chemicals, while pink dots are key targets. The size of the edges are docking scores, and the size of the dots are node degrees.

298

\section{Conclusions}

Tartary buckwheat has a very high nutritional value and is of great medicinal value to treat T2D, hypertension, and hyperlipidemia. Our studies investigated the relationships between TB and the three diseases using network pharmacology. In total, 97 putative targets were obtained from 20 composite components of TB. The TB-composite compound-putative target-known therapeutic target networks reveals that 28 key targets play a significant role in their interplay. To further study the relationships and underlying mechanisms between the key targets and pathways, key targetpathway networks were constructed. ATK2, IKBKB, RAF1, CHUK, TNF, JUN, and PRKCA were mainly involved in fluid shear stress and the atherosclerosis pathways, pathways in cancer, and the PI3K-Akt signaling pathway. Moreover, 174 candidate molecular analog docking results were obtained based on the calculation of chemical molecules from the molecular analog docking experiment. These results provide strong evidence that TB is a potential treatment to T2D, HL and HT, and that this comprehensive systemic approach integrating drug target prediction, network analysis, and molecular docking simulation is a useful tool to reveal relationships and mechanisms between the putative targets in TB and T2D, HT, and HL.

\section{References}

Ajala O, English P, and Pinkney J. 2013. Systematic review and meta-analysis of different dietary approaches to the management of type 2 diabetes. American Journal of Clinical Nutrition 97:505-516.

Andersen DK, Korc M, Petersen GM, Eibl G, Li D, Rickels MR, Chari ST, and Abbruzzese JL. 2017. Diabetes, Pancreatogenic Diabetes, and Pancreatic Cancer. Diabetes 66:1103-1110. 10.2337/db16-1477 
Brenesel MĐ, Popović T, Pilija V, Arsić A, Milić M, Kojić D, Jojić N, and Milić N. 2013. Hypolipidemic and antioxidant effects of buckwheat leaf and flower mixture in hyperlipidemic rats. Bosnian journal of basic medical sciences / Udruzenje basicnih mediciniskih znanosti = Association of Basic Medical Sciences 13:100-108.

Brown KR, and Jurisica I. 2005. Online predicted human interaction database. Bioinformatics 21:2076-2082.

Campbell CG. 1997. Buckwheat (Fagopyrum esculentum Moench.) Promoting the conservation and use of underutilized and neglected crops. Institute of Plant Genetics and Crop Plant Research,Gatersleben/International Plant Genetic Resources Institute, Rome, Italy.

Chatraryamontri A. 2010. MINT: the Molecular INTeraction database. Nucleic Acids Research 40:D857-D861.

Chen JY. 2009. HAPPI: an online database of comprehensive human annotated and predicted protein interactions. BMC genomics 10:S16.

Cheng C, Tempel D, van Haperen R, van der Baan A, Grosveld F, Daemen MJAP, Krams R, and de Crom R. 2006. Atherosclerotic Lesion Size and Vulnerability Are Determined by Patterns of Fluid Shear Stress. Circulation 113:2744-2753. 10.1161/circulationaha.105.590018

Chong H, Lee J, and Guan KL. 2001. Positive and negative regulation of Raf kinase activity and function by phosphorylation. The EMBO Journal 20:3716-3727. 10.1093/emboj/20.14.3716

Comings DE, MacMurray JP, Gonzalez N, Ferry L, and Peters WR. 1999. Association of the serotonin transporter gene with serum cholesterol levels and heart disease. Molecular genetics and metabolism 67:248-253. 10.1006/mgme.1999.2870

Desjarlais M, Dussault S, Dhahri W, Mathieu R, and Rivard A. 2017. MicroRNA-150 Modulates Ischemia-Induced Neovascularization in Atherosclerotic Conditions. Arteriosclerosis, thrombosis, and vascular biology 37:900-908. 10.1161/atvbaha.117.309189

Fabjan N, Rode J, Košir IJ, Wang Z, Zhang Z, and Kreft I. 2003. Tartary Buckwheat (Fagopyrum tataricum Gaertn.) as a Source of Dietary Rutin and Quercitrin. Journal of Agricultural and Food Chemistry 51:6452-6455. $10.1021 / \mathrm{jf034543e}$

Gorla-Bajszczak A, Juge-Aubry C, Pernin A, Burger AG, and Meier CA. 1999. Conserved amino acids in the ligandbinding and $\tau \mathrm{i}$ domains of the peroxisome proliferator-activated receptor $\alpha$ are necessary for heterodimerization with RXR. Molecular \& Cellular Endocrinology 147:37.

Gu J, Gui Y, Chen L, Yuan G, Lu H-Z, and Xu X. 2013. Use of Natural Products as Chemical Library for Drug Discovery and Network Pharmacology. PLoS ONE 8:e62839. 10.1371/journal.pone.0062839

Hegab Z, Gibbons S, Neyses L, and Mamas MA. 2012. Role of advanced glycation end products in cardiovascular disease. World Journal of Cardiology 4:90-102.

Hers I, Vincent EE, and Tavaré JM. 2011. Akt signalling in health and disease. Cellular signalling 23:1515-1527. 10.1016/j.cellsig.2011.05.004

Hopkins AL. 2007. Network pharmacology. Nature Biotechnology 25:1110.

$\mathrm{Hu}$ Y, Cheng L, Hochleitner BW, and Xu Q. 1997. Activation of mitogen-activated protein kinases (ERK/JNK) and AP-1 transcription factor in rat carotid arteries after balloon injury. Arteriosclerosis, thrombosis, and vascular biology 17:2808-2816. 10.1161/01.atv.17.11.2808

Jiansheng Li PZ, Ya Li, Yange Tian, Yonghua Wang. 2015. Systems pharmacology-based dissection of mechanisms 
357

358

359

360

361

362

363

364

365

366

367

368

369

370

371

372

373

374

375

376

377

378

379

380

381

382

383

384

385

386

387

388

389

390

391

392

393

394

395

of Chinese medicinal formula Bufei Yishen as an effective treatment for chronic obstructive pulmonary disease. Scientific Reports 5:15290.

Jishnu D, and Yu H. 2012. HINT: High-quality protein interactomes and their applications in understanding human disease. BMC Systems Biology 6:92.

Jisoon Im, And HEH, §, and Fuhung Hsieh. 2003. Effects of Processing Conditions on the Physical and Chemical Properties of Buckwheat Grit Cakes. Journal of Agricultural \& Food Chemistry 51:659-666.

Kanehisa M, and Goto S. 1999. KEGG: Kyoto Encyclopedia of Genes and Genomes. Nucleic Acids Research 27:29$34(26)$.

Katanotoki A, Satoh T, Tomaru T, Yoshino S, Ishizuka T, Ishii S, Ozawa A, Shibusawa N, Tsuchiya T, and Saito T. 2013. THRAP3 interacts with HELZ2 and plays a novel role in adipocyte differentiation. Molecular Endocrinology 27:769-780.

Kathiresan S, Melander O, Anevski D, Guiducci C, Burtt NP, Roos C, Hirschhorn JN, Berglund G, Hedblad B, Groop L, Altshuler DM, Newton-Cheh C, and Orho-Melander M. 2008. Polymorphisms associated with cholesterol and risk of cardiovascular events. The New England journal of medicine 358:1240-1249. 10.1056/nejmoa0706728

Keshava Prasad TS, Goel R, Kandasamy K, Keerthikumar S, Kumar S, Mathivanan S, Telikicherla D, Raju R, Shafreen B, and Venugopal A. 2009. Human Protein Reference Database--2009 update. Nucleic Acids Research 37:D767-772.

Kolb R, Sutterwala FS, and Zhang W. 2016. Obesity and cancer: inflammation bridges the two. Current opinion in pharmacology 29:77-89. 10.1016/j.coph.2016.07.005

Kreft M. 2016. Buckwheat phenolic metabolites in health and disease. Nutrition Research Reviews 29:30-39. $10.1017 / \mathrm{S} 0954422415000190$

Kreft S, Martina Knapp A, and Kreft I. 1999. Extraction of Rutin from Buckwheat (Fagopyrum esculentum Moench) Seeds and Determination by Capillary Electrophoresis. Journal of Agricultural \& Food Chemistry 47:46494652.

Lan SQ, Meng YN, Li XP, Zhang YL, Song GY, and Ma HJ. 2013. Effect of consumption of micronutrient enriched wheat steamed bread on postprandial plasma glucose in healthy and type 2 diabetic subjects. Nutrition Journal 12:64.

Laurent G, de Boer VC, Finley LW, Sweeney M, Lu H, Schug TT, Cen Y, Jeong SM, Li X, and Sauve AA. 2013. SIRT4 represses peroxisome proliferator-activated receptor $\alpha$ activity to suppress hepatic fat oxidation. Molecular \& Cellular Biology 33:4552-4561.

Law V. 2014. DrugBank 4.0: shedding new light on drug metabolism. Nucleic Acids Research 42:D1091.

Lee CC, Hsu WH, Shen SR, Cheng YH, and Wu SC. 2012. Fagopyrum tataricum (buckwheat) improved high-glucoseinduced insulin resistance in mouse hepatocytes and diabetes in fructose-rich diet-induced mice. Experimental Diabetes Research 2012:375673.

Lee CC, Lee BH, and Lai YJ. 2015. Antioxidation and antiglycation of Fagopyrum tataricum ethanol extract. Journal of Food Science \& Technology 52:1110.

Lee DG, Jang IS, Yang KE, Yoon SJ, Baek S, Lee JY, Suzuki T, Chung KY, Woo SH, and Choi JS. 2016. Effect of rutin from tartary buckwheat sprout on serum glucose-lowering in animal model of type 2 diabetes. Acta

PeerJ reviewing PDF | (2017:07:19272:2:0:NEW 12 Oct 2017) 
Pharmaceutica 66:297.

Li S, Zhang ZQ, Wu LJ, Zhang XG, Li YD, and Wang YY. 2007. Understanding ZHENG in traditional Chinese medicine in the context of neuro-endocrine-immune network. Iet Systems Biology 1:51.

Lin RF. 1994. Buckwheat in China. Agriculture Publishing House,Beijing. p 226-243.

Liu J, Ibi D, Taniguchi K, Lee J, Herrema H, Akosman B, Mucka P, Salazar Hernandez MA, Uyar MF, Park SW, Karin M, and Ozcan U. 2016a. Inflammation Improves Glucose Homeostasis through IKK $\beta$-XBP1s Interaction. Cell 167:1052-1066.e1018. 10.1016/j.cell.2016.10.015

Liu Z, Guo F, Wang Y, Li C, Zhang X, Li H, Diao L, Gu J, Wang W, and Li D. 2016b. BATMAN-TCM: a Bioinformatics Analysis Tool for Molecular mechANism of Traditional Chinese Medicine. Scientific Reports 6:21146.

Luo F, Gu J, Chen L, and Xu X. 2014a. Systems pharmacology strategies for anticancer drug discovery based on natural products. Molecular BioSystems 10:1912-1917.

Luo F, Gu J, Zhang X, Chen L, Cao L, Li N, Wang Z, Xiao W, and Xu X. 2014b. Multiscale Modeling of Druginduced Effects of ReDuNing Injection on Human Disease: From Drug Molecules to Clinical Symptoms of Disease. Scientific Reports 5:10064.

Luo W. 2013. Pathview: an R/Bioconductor package for pathway-based data integration and visualization. Bioinformatics 29:1830-1831.

Maldonado-Ruiz R, Montalvo-Martínez L, Fuentes-Mera L, and Camacho A. 2017. Microglia activation due to obesity programs metabolic failure leading to type two diabetes. Nutrition \& diabetes. p e254.

Malek AM, Alper SL, and Izumo S. 1999. Hemodynamic shear stress and its role in atherosclerosis. JAMA 282:20352042. 10.1001/jama.282.21.2035

Mercurio F, Zhu H, Murray BW, Shevchenko A, Bennett BL, Li J, Young DB, Barbosa M, Mann M, Manning A, and Rao A. 1997. IKK-1 and IKK-2: cytokine-activated IkappaB kinases essential for NF-kappaB activation. Science (New York, NY) 278:860-866. 10.1126/science.278.5339.860

Merendino N, Molinari R, Costantini L, Mazzucato A, Pucci A, Bonafaccia F, Esti M, Ceccantoni B, Papeschi C, and Bonafaccia G. 2014. A new "functional" pasta containing tartary buckwheat sprouts as an ingredient improves the oxidative status and normalizes some blood pressure parameters in spontaneously hypertensive rats. Food \& Function 5:1017.

Mukherjee R, Jow L, Croston GE, and Jr PJ. 1997. Identification, characterization, and tissue distribution of human peroxisome proliferator-activated receptor (PPAR) isoforms PPARgamma2 versus PPARgamma1 and activation with retinoid X receptor agonists and antagonists. Journal of Biological Chemistry 272:8071-8076.

Ohsako T YK, Ohnishi O. . 2002. Two new Fagopyrum (Polygonaceae) species, F. gracilipedoides and F. jinshaense from Yunnan, China. Genes \& genetic systems 77(6): 399-408.

Osborn O, Brownell SE, Sanchez-Alavez M, Salomon D, Gram H, and Bartfai T. 2008. Treatment with an Interleukin 1 beta antibody improves glycemic control in diet-induced obesity. Cytokine 44:141-148. https://doi.org/10.1016/j.cyto.2008.07.004

Park SH, Choi HJ, Yang H, Do KH, Kim J, Lee DW, and Moon Y. 2011. Endoplasmic reticulum stress-activated C/EBP homologous protein enhances nuclear factor-kappaB signals via repression of peroxisome proliferator-activated receptor gamma. Journal of Biological Chemistry 285:35330. 
Prakash S, and Deshwal S. 2013. Alpha and beta amylase activity of Fagopyrum esculentum (Buckwheat): A medicinal plant. Janaki Medical College Journal of Medical Science 1(1): 53-58.

Qiu J, Liu Y, Yue Y, Qin Y, and Li Z. 2016a. Dietary tartary buckwheat intake attenuates insulin resistance and improves lipid profiles in patients with type 2 diabetes: a randomized controlled trial. Nutrition Research 36.

Qiu J, Liu Y, Yue Y, Qin Y, and Li Z. 2016b. Dietary tartary buckwheat intake attenuates insulin resistance and improves lipid profiles in patients with type 2 diabetes: a randomized controlled trial. Nutrition Research 36:1392-1401.

Roy B. 2013. Biomolecular basis of the role of diabetes mellitus in osteoporosis and bone fractures. World Journal of Diabetes 4:101-113.

Scott AF, Amberger J, Brylawski B, and Mckusick VA. 2000. OMIM: Online Mendelian Inheritance in Man: Johns Hopkins University Press.

Shannon P, Markiel A, Ozier O, Baliga NS, Wang JT, Ramage D, Amin N, Schwikowski B, and Ideker T. 2003. Cytoscape: A Software Environment for Integrated Models of Biomolecular Interaction Networks. Genome Research 13:2498-2504.

Singaraja RR, Brunham LR, Visscher H, Kastelein JJP, and Hayden MR. 2003. Efflux and atherosclerosis: the clinical and biochemical impact of variations in the ABCA1 gene. Arteriosclerosis, thrombosis, and vascular biology 23:1322-1332. 10.1161/01.atv.0000078520.89539.77

Stark C, Breitkreutz BJ, Chatraryamontri A, Boucher L, Oughtred R, Livstone MS, Nixon J, Van AK, Wang X, and Shi X. 2011. The BioGRID Interaction Database: 2011 update. Nucleic Acids Research 43:D470.

Szklarczyk D, Franceschini A, Kuhn M, Simonovic M, Roth A, Minguez P, Doerks T, Stark M, Muller J, and Bork P. 2011. The STRING database in 2011: functional interaction networks of proteins, globally integrated and scored. Nucleic Acids Research 39:D561-D568.

Tang J, and Dong Q. 2017. Knockdown of TREM-1 suppresses IL-1 $\beta$-induced chondrocyte injury via inhibiting the NF- $\kappa \mathrm{B}$ pathway. Biochemical and biophysical research communications 482:1240-1245. 10.1016/j.bbrc.2016.12.019

Tong HL, Tian YP, Wang DQ, and Dong ZN. 2006. Role of tartarian buckwheat shell extract in regulation of blood lipid in rats with hyperlipidemia. Journal of the Fourth Military Medical University 27:120-122.

Ushida Y, Matsui T, Tanaka M, Matsumoto K, Hosoyama H, Mitomi A, Sagesaka Y, and Kakuda T. 2008. Endothelium-dependent vasorelaxation effect of rutin-free tartary buckwheat extract in isolated rat thoracic aorta. Journal of Nutritional Biochemistry 19:700-707.

Vida Skrabanja, §, Ivan Kreft A, and Björck† IME. 2001. Nutritional Properties of Starch in Buckwheat Products: Studies in Vitro and in Vivo. Journal of Agricultural \& Food Chemistry 49:490.

Westermeier F, Salomón C, González M, Puebla C, Guzmán-Gutiérrez E, Cifuentes F, Leiva A, Casanello P, and Sobrevia L. 2011. Insulin Restores Gestational Diabetes Mellitus-Reduced Adenosine Transport Involving Differential Expression of Insulin Receptor Isoforms in Human Umbilical Vein Endothelium. Diabetes 60:1677-1687. 10.2337/db11-0155

Wieslander G. 1996. Review on buckwheat allergy. Allergy 51:661-665.

Xenarios I, Salwínski L, Duan XJ, Higney P, Kim SM, and Eisenberg D. 2002. DIP, the Database of Interacting Proteins: a research tool for studying cellular networks of protein interactions. Nucleic Acids Research 
474

475

476

477

478

479

480

30:303-305.

Yao B, Zhang Y, Delikat S, Mathias S, Basu S, and Kolesnick R. 1995. Phosphorylation of Raf by ceramide-activated protein kinase. Nature 378:307-310. 10.1038/378307a0

Yu G, Wang LG, Han Y, and He QY. 2012. clusterProfiler: an R Package for Comparing Biological Themes Among Gene Clusters. Omics : a journal of integrative biology 16:284.

Yu W, Xin F, Jing X, Wang Q, and Kuang H. 2016. Systems pharmacology to investigate the interaction of berberine and other drugs in treating polycystic ovary syndrome. Scientific Reports 6:28089.

Zhang X, Zhang S, Yamane H, Wahl R, Ali A, Lofgren JA, and Kendall RL. 2006. Kinetic mechanism of AKT/PKB enzyme family. The Journal of biological chemistry 281:13949-13956. 10.1074/jbc.m601384200

Zhang Y, Xia M, Guo Q, Ming B, Bo Z, Liu C, Sun Y, Shao L, and Na L. 2016. Pathway of PPAR-gamma coactivators in thermogenesis: a pivotal traditional Chinese medicine-associated target for individualized treatment of rheumatoid arthritis. Oncotarget 7:15885-15900.

Zhang YW, Gesmonde J, Ramamoorthy S, and Rudnick G. 2007. Serotonin transporter phosphorylation by cGMPdependent protein kinase is altered by a mutation associated with obsessive compulsive disorder. Journal of Neuroscience the Official Journal of the Society for Neuroscience 27:10878.

Zhang ZL, Zhou ML, Tang Y, Li FL, Tang YX, Shao JR, Xue WT, and Wu YM. 2012. Bioactive compounds in functional buckwheat food. Food Research International 49:389-395.

Zhou Y, Li B, Cui L, Zhou X, and Ning L. 2015. The Effect of Tartary Buckwheat Resistant Starch on Physiological Function of Diabetic Mice. Journal of the Chinese Cereals \& Oils Association 30:24-28.

\section{Supplemental Information}

Supplementary Table S1: Information about the composite compounds of Fagopyrum tataricum.

Supplementary Table S2: Known therapeutic targets for Type II diabetes.

Supplementary Table S3: Known therapeutic targets for hypertension.

Supplementary Table S4: Known therapeutic targets for hyperlipidemia.

Supplementary Table S5: Detailed information on eight existing protein-protein interaction databases.

Supplementary Table S6: Prediction of putative targets for composite compounds of Fagopyrum tataricum.

Supplementary Table S7: Overlap of significant targets in PT, T2D, HT, and HL.

Supplementary Table S8: Four topological feature values of key targets in the TB-composite compound-putative target-known therapeutic target network. 
507 Supplementary Table S9: Four topological feature values of key targets in the target-pathway 508 network.

509 Supplementary Table S10: Detailed information on the pathway of key targets.

510 Supplementary Table S11: Detailed docking information on key targets and composite 511 compounds.

512 Supplementary Table S12: Four topological feature values of targets in the target-composite 513 compound network. 
Figure 1

Figure 1. Venn diagram showing the overlap of significant targets in PT, T2D, HT, and $\mathrm{HL}$.

PT=putative targets, green; T2D = type II diabetes, blue; HT=hypertension, pink; and $\mathrm{HL}=$ hyperlipidemia, yellow.

\section{T2D}

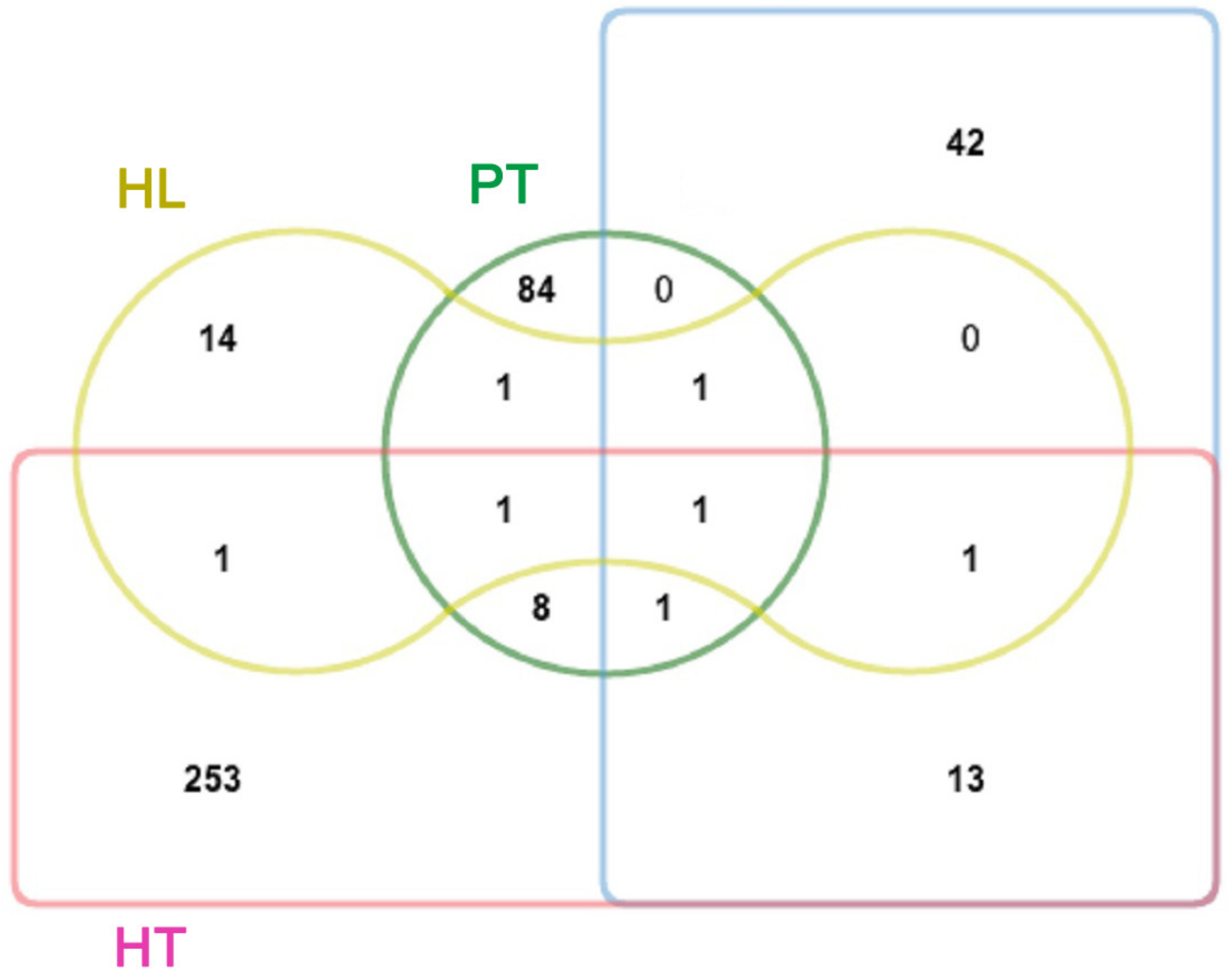


Figure 2

Figure 2. Interaction network between chemical components of TB, their putative targets, and known therapeutic targets of the three diseases built and visualized with Cytoscape.

Blue line: linked PT and their targets; purple: linked T2D and their targets; green: linked HL and their targets; yellow: linked HT and their targets; and light blue: linked chemical components and their targets.

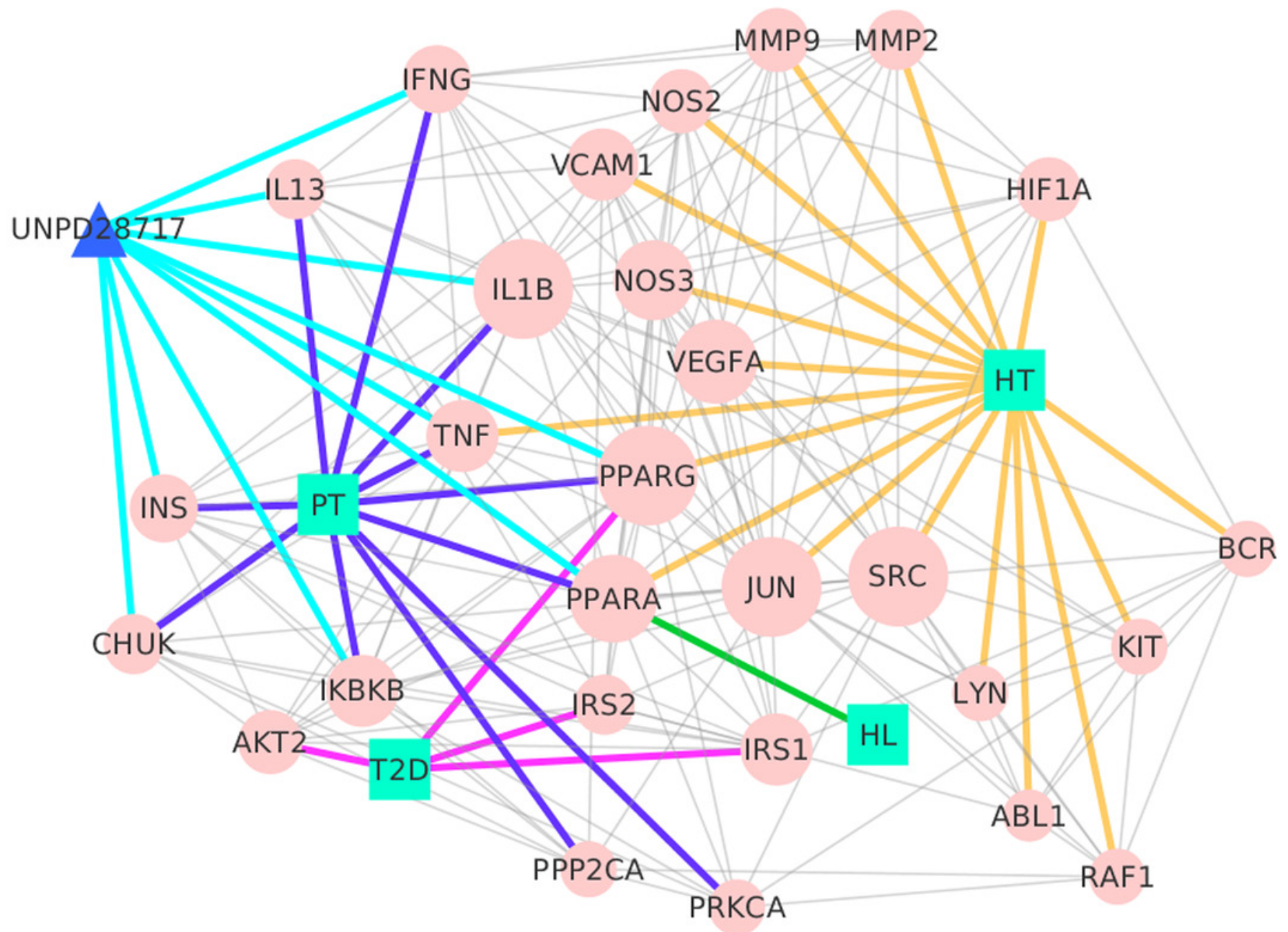




\section{Figure 3}

Figure 3. The target-pathway network.

Pink dots are targets, purple diamonds are pathways, and the dot size and diamond size represent node degree value.

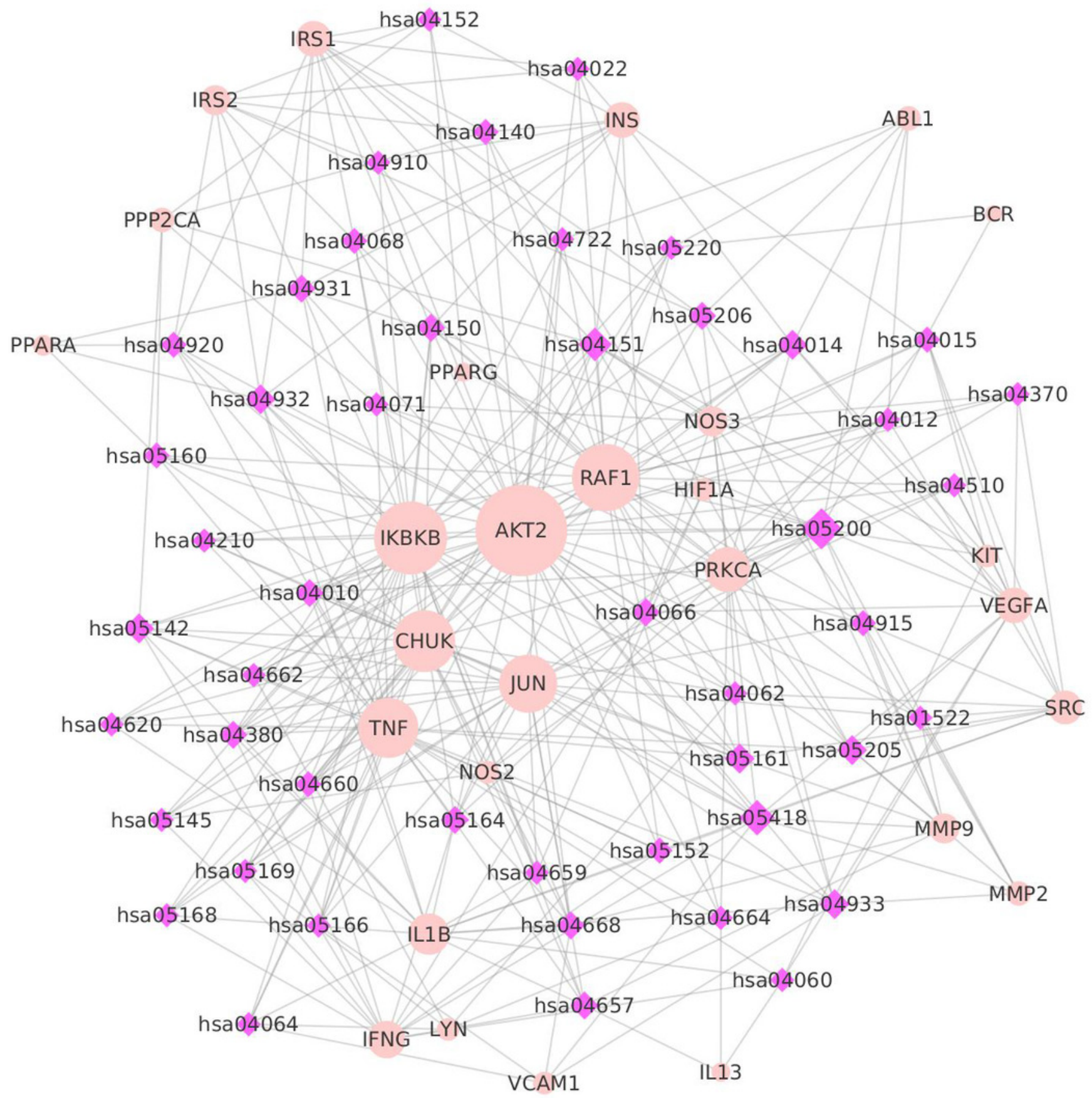




\section{Figure 4}

Figure 4. The target-composite compound network. Blue dots are chemicals, while pink dots are key targets.

The size of the edges are docking scores, and the size of the dots are node degrees.

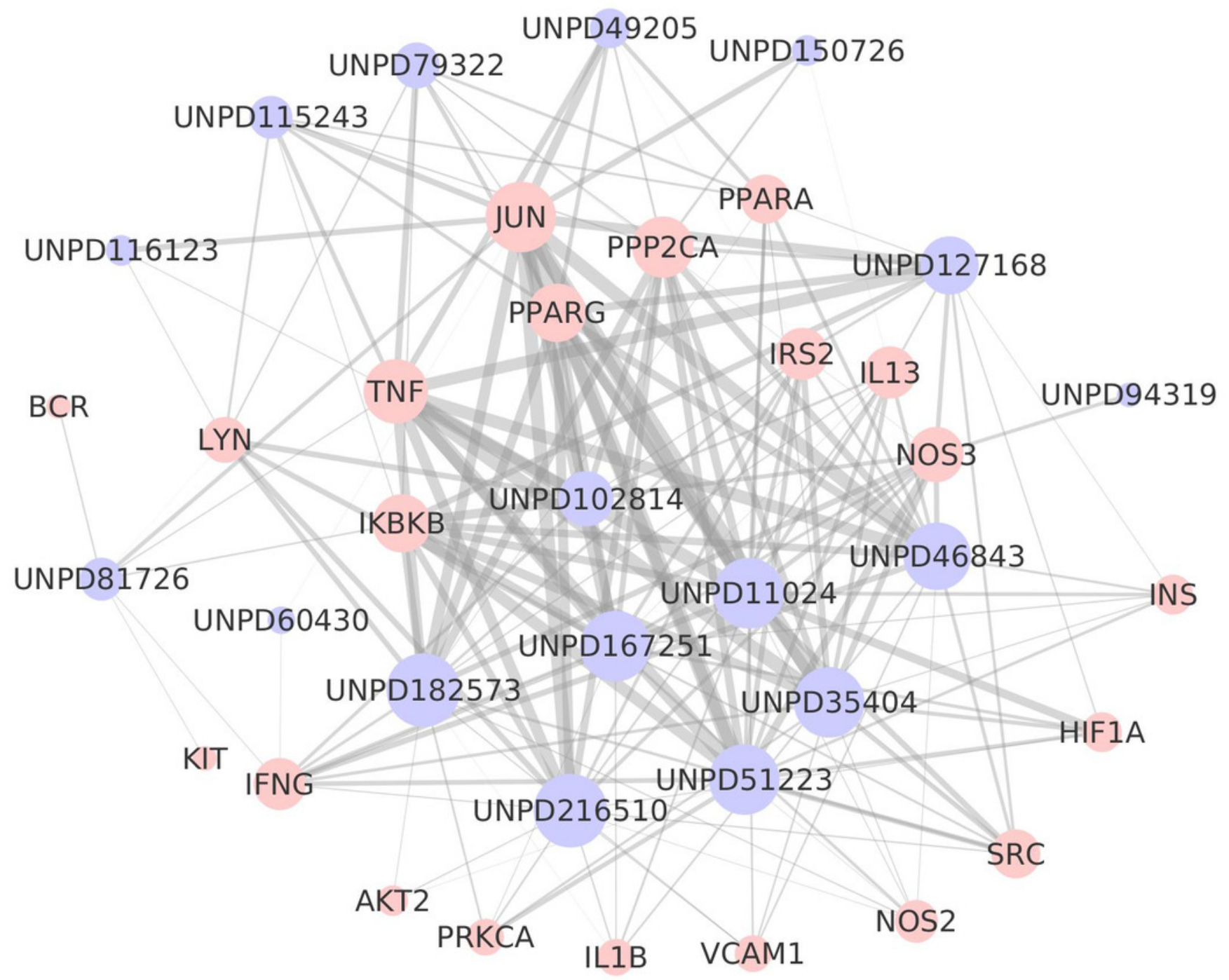

